International Journal of Medical Anesthesiology 2020; 3(1): 120-121

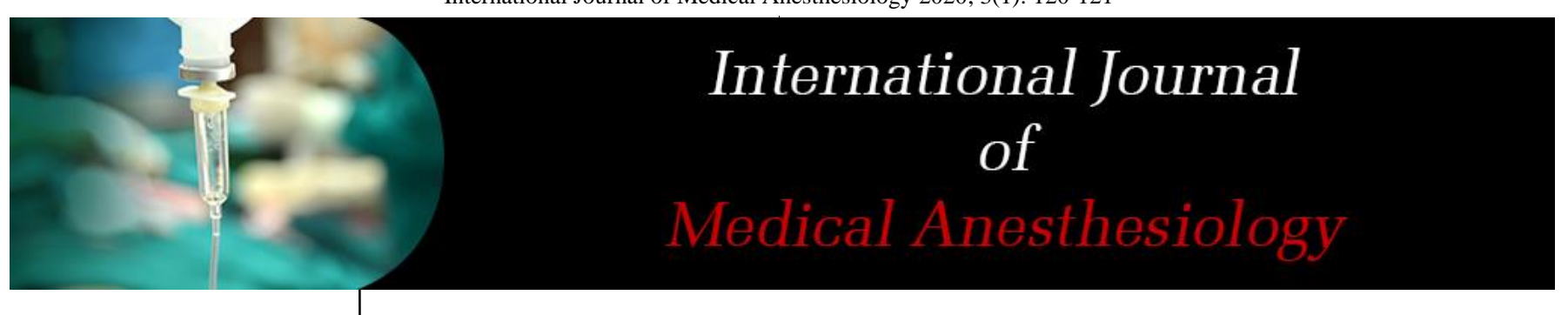

E-ISSN: 2664-3774

P-ISSN: 2664-3766

www.anesthesiologypaper.com

IJMA 2020; 3(1): 120-121

Received: 21-11-2019

Accepted: 25-12-2019

\section{Priyanka Singh}

Senior Resident, Department of Anesthesiology, ABVIMS, Dr. RML Hospital, Delhi, India

\section{Shruti Sharma}

Senior Resident, Department of Anesthesiology, ABVIMS, Dr. RML Hospital, Delhi, India
Corresponding Author: Priyanka Singh Senior Resident, Department of Anesthesiology, ABVIMS, Dr. RML Hospital, Delhi, India

\section{Anaesthesia for pathological fracture fixation in a patient of multiple myeloma with CKD on hemodialysis}

\section{Priyanka Singh and Shruti Sharma}

DOI: https://doi.org/10.33545/26643766.2020.v3.i1b.79

\section{Abstract}

Multiple myeloma is a neoplastic proliferation of plasma cells mostly affecting aged people. Common manifestations are increased susceptibility to infection, lytic lesions in bone, renal failure, hemorrhagic tendencies, hyper viscosity syndrome, anemia and hypercalcemia. We report a case of 35 year female of Multiple myeloma with bone involvement posted for fixation of pathological fracture femur, discussing the perioperative considerations and management for anesthesiologists.

Keywords: Multiple myeloma, central neuraxial blockade, hemodialysis

\section{Introduction}

Despite the relatively common frequency, there is a paucity of information about the delivery of anaesthesia for orthopedic oncologic surgeries. Multiple myeloma per se incidence is $13 \%$ of all hematological malignancies [1]. There are pre-operative, intraoperative and postoperative concerns specific to these patients and procedures. The major challenge of modern myeloma management is, optimizing quality of life with effective supportive care.

\section{Case report}

A 35 year woman, weighing $50 \mathrm{~kg}$ presented to the hospital with pain in left lower limb. She has been diagnosed with multiple myeloma one year ago for which she underwent three cycles of chemotherapy and had bone involvement as well as nephropathy. X ray left lower limb showed pathological fracture of left shaft of femur requiring intramedullary nailing of left shaft of femur. She underwent 2 cycles of chemotherapy in the hospital including lenalidomide + dexamethasone +bortezomib. She had also undergone dialysis twice in view of deranged renal function tests. Post dialysis blood investigations were Hemoglobin9.2gm\%, TLC-2100, Platelet count-1.62lacs, PT/INR-14.7/1.34, blood urea-43, serum craetinine-2.1, Uric Acid-3.8, serum sodium -133, s.potassium-3.2, Ca-8.7, RBS-128. ECG and Chest $\mathrm{x}$ ray were normal. We have advised for a skeletal survey and 2D Echocardiography. Post chemotherapy 2D echo appeared to be normal. USG color Doppler for left lower limb was done to rule out any venous thrombosis.

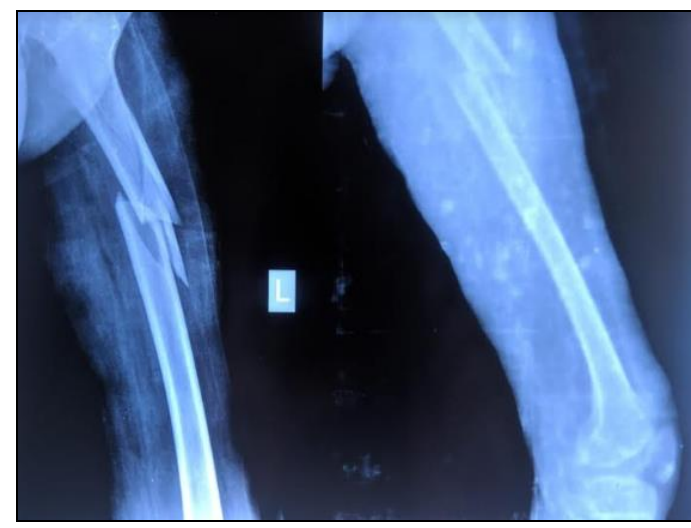

Fig 1: X-ray left femur showing pathological fracture of the shaft 
A wide bore intravenous cannula was secured and the patient was hydrated with $10 \mathrm{ml} / \mathrm{kg}$ of ringer lactate before induction of anesthesia. Preoperative antibiotic prophylaxis was administered with injection ceftriaxone $1 \mathrm{gm}$ intravenous. Her preoperative hemodynamic parameters were within normal range i.e. PR-84/min, BP-110/68mmhg, spo2-99\% on room air. The case was decided to be done under central neuraxial blockade (Combined spinal epidural). Patient was put on low molecular weight heparin which was stopped 24 hour before surgery.

Patient was made to lie in sitting position and under all aseptic precautions, epidural space was identified in L2-L3 space. $2.5 \mathrm{cc}$ of inj. bupivacaine $0.5 \%(\mathrm{H})$ with inj. fentanyl $0.5 \mathrm{ml} 25 \mathrm{mcg}$ as an adjuvant was given intrathecally through the same space, after which an epidural catheter was placed in situ for post-operative pain relief. Patient was made to lie in supine position with leftward tilt immediately for unilateral anaesthesia. After ten minutes patient was positioned in right lateral for surgery to be started. The procedure was uneventful with no hemodynamic disturbance and minimal/allowable blood loss. Post operatively, patient was comfortable with infusion of $8 \mathrm{ml}$ of $0.0625 \%$ bupivacaine given epidurally. She did not require any rescue analgesics. Renal function test and s. calcium were normal in post-operative period. Patient was discharged on fifth postoperative day.

\section{Discussion}

Multiple myeloma, also called as kahler's disease is a neoplastic proliferation of plasma cells in bone marrow derived from a single clone. Multiple myeloma accounts for $13 \%$ of all hematologic malignancies in whites and $33 \%$ in blacks with an incidence of 1-9 per 100,000 worldwide ${ }^{[2]}$. The clinical manifestations of symptomatic Multiple myeloma are lytic or osteopenic bone lesions, hypercalcemia, renal failure, anemia and infections, but patients are now diagnosed earlier at an asymptomatic stage [3]. These lytic lesions are due to clonal proliferation of malignant plasma cells that accumulate in bone marrow, causing bone destructions and marrow failure presented as pathological fractures in patients. Due to number of organ dysfunctions associated, it demands considerable attention perioperative while managing such patients.

Kidney injury is a common complication of Multiple myeloma and other plasma cell dyscrasias. Defined by abnormal Creatinine clearance, renal insufficiency is present in nearly one half of myeloma patients at presentations and associated with increased mortality ${ }^{[4]}$. Adequate hydration, correction of hypercalcemia and hyperurecemia and antimyeloma therapy should be initiated promptly. As this patient is in renal failure requiring dialysis, it was done preoperatively as well for optimization of renal function tests. The post dialysis RFTs and s. calcium were came to normal range. We ensured adequate hydration by co loading/preloading with iv fluids.

Hemodynamics were maintained intraoperatively and an epidural catheter was placed for post-operative pain relief, thus avoiding use of NSAIDS and preventing deterioration of renal functions in perioperative period, risk of renal failure and improving long term survival ${ }^{[2]}$. After introduction of newer chemotherapeutic agents, the survival rate is improved in these patients, but subjected them to increased risk of developing adverse events. Drugs such as lenalidomide and botezomib have serious side effects, which is one of the concerns in perioperative period.

Central neuraxial blockade (CNB) and regional nerve block can be performed safely in these patients. Bridging the patient to low molecular weight heparin from antiplatlet and anticoagulants in perioperative period helps anesthesiologists to perform CNB without risk of bleeding or thromboembolism (subjected to normal coagulation profile). A case of successful management of caesarean section under $\mathrm{CNB}$ has been reported earlier ${ }^{[5]}$. Also, managing patient of multiple myeloma for lower limb surgery with combined sciatic psoas compartment nerve block is reported ${ }^{[6]}$. These are done to avoid intraoperative hemodynamic changes. Fanelli et al., [7] found better hemodynamic outcome in patients who underwent combined sciatic femoral block or unilateral spinal anaesthesia than general anaesthesia.

We decided to give a comparative low dose of local anaesthetic agent along with an adjuvant intrathecally for providing unilateral spinal anesthesia and placing an epidural catheter to provide intraoperative and postoperative pain relief, thus avoiding use of NSAIDS. A multimodal approach is to be used for pain management in such patients.

\section{Conclusion}

A thorough preoperative evaluation should be done for significant co morbidities and to ensure optimization prior to procedure. These surgeries are more complex and have potential for massive blood loss and hemodynamic instability. The use of regional anaesthesia/ peripheral or neuraxial block as a sole anaesthetic may be effective alone or in combination with GA. Post-operative plan for thromboprophylaxis must be defined prior to choosing the anaesthetic technique. Pain control requires multimodal approach. Well formulated anaesthetic plan, effective communication between anesthesiologist and surgeons, ensures optimal patient outcomes.

\section{Conflict of interest - None}

\section{References}

1. Palumbo A, Anderson K. Multiple Myeloma. $N$ Engl J Med. 2011; 364:1046-60.

2. Bisoyi S, Narayan Pratihary B, Mohapatra R. Perioperative considerations in the management of a patient with multiple myeloma undergoing aortic valve replacement. J cardiothoracic vascular anaesthesia. 2015; 29:151-5.

3. Röllig C, Knop S, Bornhäuser M. Multiple myeloma, Lancet. 2015; 385:2197-208.

4. Kidney disease and multiple myeloma M.cjasn.asnjournals.org, 2013.

5. Dabrowska DM, Gore C, Griffith S, Mudzingwa S, Varaday S. Anaesthetic management of a pregnant patient with multiple myeloma. Int J Obstet Anaesth. 2010; 19:336-7.

6. Binici O, Akyoi F. Combined sciatic psoas compartment nerve block in a patient with multiple myeloma. East J Med. 2016; 21:197-99.

7. Fanelli G, Casati A, Aldegheri G et al. Cardiovascular effects of two different regional anaesthetic techniques for unilateral leg surgeries. Acta Anaesthesiol Scand. 1998; 42:80-84.s 